\title{
Hierarchical Bayesian approach applied to the formulation of sustained-release suppositories and dissolution profile modeling
}

\author{
Abdelhafid Benomar (D), Casimir Adade Adade* (D), Yassir Elalaoui, Naoual cherkaoui, Younes Rahali, Abdelkader laatiris, \\ Aicha Fahry \\ Team of Formulation and Quality Control of Health Products, Laboratory of Pharmaceutics, Faculty of Medicine and Pharmacy, Mohammed V University of \\ Rabat, Rabat, Morocco.
}

\begin{tabular}{l}
\hline ARTICLE INFO \\
\hline Received on: $28 / 02 / 2021$ \\
Accepted on: $12 / 04 / 2021$ \\
Available online: $05 / 07 / 2021$
\end{tabular}

\section{Key words:}

Drug formulation, sustainedrelease, drug release, kinetics modeling, suppository, widely applicable information criterion, leave-one-out.

\begin{abstract}
The hierarchical Bayesian modeling approach was used to select the appropriate empirical kinetics model of sustained release and to optimize the in vitro dissolution rate of the sustained-release suppository by controlling the composition of Eudragit L-100 and Eudragit S-100 in the experimental mixture. Thirteen formulations of suppositories were prepared with $2 \mathrm{~g}(10 \%)$ mixture of Eudragit ${ }^{\circledR} \mathrm{R}-100$ and S-100 according to a personalized mixture experimental design. The cumulative release of active ingredient was measured at five times (20, 50, 80, 160, and 235 minutes).

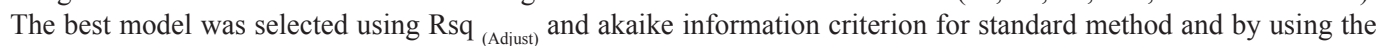
weight of widely applicable information criterion (WAIC) and leave-one-out (LOO) cross-validation for the Bayesian approach. Frequentist approach gave three best model depending on the formulation. Compared to this, the Bayesian method was able to define a single model, which is the first-order model. The relative probability of this model is 0.97, 0.99 based on the WAIC, and LOO, respectively. The relationship between $K_{1}$ (Release rate constant) and the quantities of the two Eudragits is quadratic, for Eudragit_L, $Q_{\text {release }}(\%)=0.0031 X^{2}-0.0026 X+0.0069$ and $X$ is the Eudragit L100 and $K_{1}$ (Rate release) $=0.41$ minutes $^{-1}$. The Bayesian method allowed finding the most adequate model among several models that can be generated by the standard frequentist approach.
\end{abstract}

\section{INTRODUCTION}

Mathematical models play a crucial role in the interpretation of drug release mechanism (Gouda et al., 2017; Grassi et al., 2006; Paarakh et al., 2018; Varma et al., 2004). The drug release kinetics for given formulations has been usually fitted to predefined semi-empirical models using non-hierarchical frequentist modelling approach. The appropriate model for each formulation is selected based on the highest Rsq (Adjust) and the lowest Akaike Information Criterion (AIC) (Gouda et al., 2017; Paarakh et al., 2018; Victor and Francis, 2017). Such a situation may lead to variation in the best-fitting model among formulations, in particular when the number of points in the curve is limited.

\section{*Corresponding Author}

Casimir Adade Adade, Team of Formulation and Quality Control of

Health Products, Laboratory of Pharmaceutics, Faculty of Medicine and

Pharmacy, Mohammed V University of Rabat, Rabat, Morocco.

E-mail: casimir.adade@gmail.com
This is not meaningful biologically given the interdependency between data and the model convergence may be problematic (Piray et al., 2019). Hierarchical Bayesian modelling can solve these issues by expressing the model over all formulations and consequently addressing both model fitting and model comparison within the same framework (Piray et al., 2019). Hierarchical Bayesian modelling approach assumes that the formulations share the same best model and differ only in some or all parameters values of the model (Andrews et al., 2002; Millar, 2009; Wiecki et al., 2013). The differences between the two statistical concepts have their root in differing conceptions of probability. In practice, frequentist approach quantify the properties of data-derived quantifies, as for Bayesian approach quantify the incertitude of measure (VanderPlas, 2014).

For pharmaceutical development, the application of Bayesian statistics remains modest and mainly affects the field of clinical trials and not the field of formulation. This will be one of the strongest points of our formulation study (Natanegara et al., 2014; Price and LaVange, 2014) . 
The use of polymers in the drug delivery system increased during the last years as a result of the advantages offered by these molecules (Andrews et al., 2002; Malekmohammadi et al., 2019; Zuo et al., 2014). For sustained-release system, Eudragits, good safety profile molecules, may give the formulator the possibility of choosing between different categories depending on the purpose of the formulation such as oral, colon, rectal, and even transdermal (Joshi, 2013; Nikam et al., 2011; Thakral et al., 2013). For clonic targeting, both Eudragit L-100 and S100 are widely used for molecule delivery as they offer muco-adhesiveness and pH-dependent release (Khan et al., 1999; Lee et al., 2020). Only one study has been reported in the scientific literature on the use of these polymers in rectal drug delivery (Baloglu et al., 2002). In this study, authors prepared and evaluated the kinetics of sustained-release suppositories of polyethylene glycol (PEG) 400, PEG 4000, Witepsol, and Eudragit L-100. In our study, the choice of these Eudragits comes from the fact that the physiological conditions of the colon (especially the distal colon) and those of the rectum were similar for the $\mathrm{pH}(\mathrm{pH}=7-8)$; hence, the interest of these polymers called $\mathrm{pH}$-Dependent Drug Delivery Systems in the formulation of Hydrophilic suppositories (Hua, 2019; Lee et al., 2020). By optimizing the mixture composition of these polymers (EL-100 and ES-100) and the total polymers content of the matrix, a specific release rate for the active molecule can be obtained by modifying the diffusivity through the gelled of the matrix structure (Di Colo et al., 2002; Misra, 2014; Nikam et al., 2011; Surti et al., 2020).

The novelty of this study is the combination of two Eudragit polymers in the formulation of sustained release suppositories and the application of the Bayesian hierarchical approach to the modeling of the release kinetics. The first objective of this study is to use the hierarchical Bayesian modeling approach to select the appropriate empirical kinetics model of Barbital sustained release. The second objective is to optimize the in vitro release rate of sustained-release suppository by controlling the composition of E-L100 and E-S100 in the experimental mixture.

\section{MATERIALS AND METHODS}

\section{Materials}

All the materials used in this study, i.e., "Sodium Barbital”, PEG 6000, Eudragit ${ }^{\circledR}$ L100, Eudragit ${ }^{\circledR}$ S100 (Rohm Pharma, GmbH, Germany), $\mathrm{NaH}_{2} \mathrm{PO}_{4}$ (monobasic), and $\mathrm{Na}_{2} \mathrm{HPO}_{4}$ (dibasic) were acquired by the galenic pharmacy laboratory of the Faculty of Medicine and Pharmacy.

\section{Design of experiments and statistical analysis}

In the present study, we used a personalized design of experiment with constraint and with process factor, which was time. The quantities of the two Eudragits varied from 0 to $2 \mathrm{~g}$ each, with upper constraint of $2 \mathrm{~g}$ for the sum of the two Eudragits L and S, based on preliminary tests. Beyond this limit, there would be little flow, poor filling of the metal molds, and an unacceptable appearance of the prepared suppositories. The quantity of Macrogol 6000 and Barbital were set to 17.4 and 0.6 $\mathrm{g}$, respectively (Table 1). For each of the 13 formulations, three experiments was made and five sampling performed at 20, 50, 80, 160 , and 235 minutes.
Table 1. Matrix of experiments with proportions of fixed (PEG 6000 and Barbital) and variable (EL-100 and ES-100) components.

\begin{tabular}{ccccc}
\hline \multirow{2}{*}{ Formulation } & \multicolumn{2}{c}{ Fixed components (\%) } & \multicolumn{2}{c}{ Variable components (\%) } \\
\cline { 2 - 5 } & PEG 6000 & Barbital & EL-100 & ES-100 \\
\hline 1 & 87 & 3 & 3.5 & 6.5 \\
2 & 87 & 3 & 10 & 0 \\
3 & 87 & 3 & 9 & 1 \\
4 & 87 & 3 & 1.5 & 8.5 \\
5 & 87 & 3 & 7.5 & 2.5 \\
6 & 87 & 3 & 6 & 4 \\
7 & 87 & 3 & 5 & 5 \\
8 & 87 & 3 & 6.5 & 3.5 \\
9 & 87 & 3 & 1 & 9 \\
10 & 87 & 3 & 2.5 & 7.5 \\
11 & 87 & 3 & 4 & 6 \\
12 & 87 & 3 & 8.5 & 1.5 \\
13 & 87 & 3 & 0 & 0 \\
\hline
\end{tabular}

\section{Preparation of suppositories by fusion molding}

The preparation consisted in melting the quantity of PEG 6000 of each formulation at $46^{\circ} \mathrm{C}$ in the stainless steel capsule, then adding the other components under agitation (risk of gelation of the mass) and without incorporation of air bubbles. The prepared suppositories was scraped and stored in glass bottles.

\section{Chemical and pharmaco-technical assay of the prepared suppositories}

The prepared suppositories have been evaluated following the USP monographs for appearance, weight variation, content uniformity of dosage form, hardness, disintegration time, and dissolution assay. The Barbital content was determined using a UV-visible spectrophotometer. The hardness was performed with the Erweka AR 400 hardness tester (Erweka Apparatebau-GmbH Germany). The disintegration was performed in a $6.8 \mathrm{pH}$ buffer solution at $37^{\circ} \mathrm{C}(+/-0.5)$ using the U.S.P. tablet disintegration apparatus (SOTAX DT 3, Heusenstamm, Germany).

\section{Dissolution testing}

The suppository was placed in the basket, lowered to the precise level in $1 \mathrm{~L}$ vials filled with $500 \mathrm{ml}$ phosphate buffer ( $\mathrm{pH} 7.2$ ), and conditioned at $37^{\circ} \mathrm{C} \pm 0.5$. The basket apparatus was set at $50 \mathrm{rpm}$. Three millilitre of the dissolution medium was withdrawn with a syringe at defined time intervals ( $t 20, \mathrm{t} 50, \mathrm{t} 80$, $\mathrm{t} 160$, and $\mathrm{t} 235$ minutes). The volume withdrawn was replaced by its equivalent in phosphate buffer. The optical density of the filtrate (filters $0.24 \mu \mathrm{m}$ ) was measured at $245 \mathrm{~nm}$ with the UVvisible spectrophotometer (Spectrophotometer 6305 UV-VIS 198 at $1000 \mathrm{~nm}$ Jenway, Bibby Scientific France SAS) (Onyeji et al., 1999; Özgüney et al., 2007; Ranjita and Kamalinder, 2010).

\section{Modeling dissolution kinetics}

For modified release suppositories, the release of the active principle is due to different parameters such as disintegration, diffusion of the active principle through a membrane, swelling, and diffusion through a polymer, erosion of a matrix (Costa and 
Lobo, 2001; Faisant et al., 2002; Varma et al., 2004). Various kinetics models were used to describe the release kinetics (Faisant et al., 2002; Peppas and Narasimhan, 2014).

The zero-order model (Equation 1) is applied to pharmaceutical forms that do not disintegrate and that release the product over a long period of time. These are of matrix modifiedrelease forms with poorly soluble active ingredients, coated forms and osmotic forms (Moodley et al., 2012). The first-order model (Equation 2) was proposed by Wagner for forms whose release depends on $\mathrm{pH}$ (Basak et al., 2008). The Higuchi model (Equation 3 ) is applicable if diffusion is the only mechanism responsible for the release of the active ingredient (Craig, 2002). The KorsmeyerPeppas model (Equation 4) is used to classify drug release from polymers as fickian and non-fickian (Güneri et al., 2004). The Hixson-Crowell model (Equation 5) describes the release of systems in which the surface area and diameter of the matrix change over time (Dash et al., 2010).

The so-called Zero Order:

$Q 0=K_{0} t$

The first-order model:

$Q 1=\left(1-e^{-k t}\right)$

The Higuchi kinetics model:

$Q_{K p}=k_{K P} t^{n}$

The Korsemyer-Peppas kinetics model:

$Q_{H}=K_{H} t^{n}$

Hixson-Crowell model

$$
Q_{H C}=\left\{1-\left(1-K_{H C} t\right)^{3}\right\}
$$

where $Q$ is the cumulative percent of drug release of Barbital, $Q_{0}$, $Q_{\mathrm{H}}, Q_{\mathrm{KP}}$, and $Q_{\mathrm{HC}}$ are the intercepts of the models, $k_{0}, K_{1}, K_{\mathrm{HC}}$, and $K_{\mathrm{KP}}$ are the rate of release, $n$ is the diffusional exponent indicating the drug-release mechanism. When the values of $n$ equal to 0.5 , the drug release, follow the Fickian diffusion law. When $0.5<n<$ 1 , the drug release follow a non-Fickian diffusion. When $n=1.0$, the drug release is caused by swelling of the polymer (Costa and Lobo, 2001; Zhang et al., 2010).

\section{Statistical analysis}

Frequentist approaches study the behaviour of the data, i.e., the probability of repetition of events. Bayesian approaches are based on the measurement of uncertainty.

\section{Frequentist approach}

Data of each Formulation were fitted individually to the five above-mentioned models using DD-Solver which is an add-in program in Microsoft Excel. DD-Solver uses the nonlinear leastsquares fitting technique, and models parameters were determined based by minimizing the sum of squares errors (Nakata, 2010; Zuo et al., 2014). The initial values of the model were set individually before the iterative optimization. The best model for each formulation is selected based on several criteria such as R-sq Adjusted and AIC. Both Rsq and AIC present a limitation in the model selection and inference process when the number of parameters are different between candidate models (Nakata, 2010).
In addition, the DD-solver does not allow for hierarchization of one or all model parameters. Given that, the selected model (bestfitting model) may vary from formulation to another in particular when the number of points in the curve is limited (Nakata, 2010).

\section{Bayesian approach}

Data of all formulations were fitted to the five above mentioned models using a hierarchical Bayesian model using the PyMC3 packages of Python (Patil et al., 2010; Salvatier et al., 2016). This approach assumes that the formulations share the same best model and differ only on values of some or all parameters of the model, for each of the five models. Prior parameters of each model were as follows: the variation was assumed zero and model parameters were set to the initial value obtained when the model was fitted to all data.

The trace plot, the posterior distribution of the parameters over each Markov chain Monte Carlo (MCMC) and the convergence of the chains were used to examine the performance of the models (Bates and Campbell, 2001; Cowles and Carlin, 1996). MCMC is a class of algorithms which can efficiently characterize even high dimensional posterior distributions through drawing of randomized samples such that the points are distributed according to the posterior. For each model, chain MCMC of 2000 cycles were considered with a burn-in period of first 200 cycles.

We used two methods for Bayesian model selection such as the widely applicable information criterion (WAIC) and Leaveone-out cross-validation (LOO) (Vehtari et al., 2017; Watanabe and Opper, 2010). The methods evaluate models based on their predictive validity. LOO method examines each left out piece of data, the performance of the model. WAIC uses the entire posterior distribution of models to assess model performance (Gelman et al., 2014; Luo and Al-Harbi, 2017; Vehtari et al., 2015). Widely available information criterion is calculated as Gelman et al. (2014) as:

$$
\mathrm{WAIC}=-21 \mathrm{ppd}+2 p_{\mathrm{WAIC} n}
$$

The lppd is the log point-wise predictive density and can be approximated as:

$$
\begin{aligned}
& \operatorname{lppd}=\sum \log \left(E_{\text {post }} \mathrm{p}(\mathrm{yi} \mid \theta)\right) \\
& \text { and } \\
& p_{\text {WAICn }}=\sum \operatorname{var}_{\text {post }} \log (\mathrm{p}(\mathrm{yi} \mid \theta))
\end{aligned}
$$

The simpler way to perform model selection is to rank models based on their WAIC or LOO value and to choose the model with the lowest one, However, this simple method do not take in to account the uncertainty of WAIC of each model given the standard error of WAIC. As such, we performed a model selection calculating an average weight for each model relative to the best model (the lowest WAIC or LOO):

$$
\text { wi }=\exp \left(-1 / 2 \mathrm{dIC}_{i}\right) / \sum_{\mathrm{j}} \mathrm{M}^{\mathrm{exp}}\left(-1 / 2 \mathrm{dIC}_{j}\right)
$$

where wi is the weight of the model $i$, which corresponds to its relative probability relative to all tested models.

For the model selection, we have five hierarchical models cited above, in addition we add the corresponding pooled models for each category of the model, which aim to test the hypothesis of the significant effect of the quantities of the two 
Eudragits on the rate of release. In other words, the selection of hierarchical model corresponds to a significant effect of Eudragits quantities on release rate; inversely the selection of pooled model corresponds to the lack of effect of Eudragits quantities on the kinetic of release.

The effect of Eudragits on the release rate was assessed by investigating the relationship between the parameters of the selected model and the weight of EL-100 or ES-100 in the formulation using the non-linear regression technique in Scipy package.

\section{RESULTS AND DISCUSSION}

All suppositories satisfy the requirement of the European pharmacopeia content uniformity, weight variation, hardness, and disintegration time. Although, the uniformity of content varied from $96.1 \%$ to $101.5 \%$, and the weight variation from 2.23 to 2.67 $\mathrm{g}$. The hardness of all formulations was superior to $4.8 \mathrm{~kg}$ and the disintegration time ranged between 30 and 203 minutes.

\section{In vitro release modeling}

In vitro dissolution tests have shown that the prepared suppositories have an sustained-release effect, even if it does not contain Eudragit (formulation No. 13 with PEG 6000 only). The percentage of Barbital dissolved in 4 hours (235 minutes) was more than $90 \%$ for four formulations (F3, F5, F12, and F13), more than $80 \%$ for formulations F2, F6, F7, and F8 and less than $80 \%$ for five formulations (F1, F4, F9, F10, and F11).

\section{Frequentist model selection approach}

In vitro release characteristics of sustained-release suppositories were evaluated to determine the best-fitted kinetic model (Table 2). The release rates of all formulations were best described with first-order model, which presented an Rsq (Adjust) $>0.90$ and $\mathrm{AIC}<16$ model (Fig. 1). Based on the $\mathrm{Rsq}_{(\text {Adjust) }}$, the first-order model was the best model for formulations $1,2,3,4$,
5, 8, and 13, the Higuchi model is best for formulation 9, the Korsmeyer-Peppas model was best for formulations 6, 10, and 11 and the Hixson-Krowell model was best for both formulations 7 and 12. Based on AIC, the first-order model was best for formulations $1,2,3,4,5,8$, and 13, Korsmeyer-Peppas is the best model for formulations 9,10 , and 11 and finally the best model for formulations 6 and 7 was Hixson-Krowell (Zhang et al., 2010; Zuo et al., 2014). This showed that the release for the same formulations can have different best models in vitro, which is one of the weaknesses of the Frequentist approach. In fact, the findings from this approach made no clear biopharmaceutical meaning.

\section{Bayesian model selection approach}

The first order model presented the lowest value of WAIC and LOO. The standard errors (SE) varied from 3 to 15. The weight of the model was 0.97 and 0.99 based on both WAIC and LOO, respectively. The relative probability (weight) of the selected model was greater than 0.5 , indicating that no competing model was possible. The LOO and WAIC estimate the same predictive performance criteria. The selection of the pooled model represented the $\mathrm{H} 0$ hypothesis, i.e., $K_{1}$ is not affected by the quantities of the two Eudragits, while the selection of the hierarchical model represented the alternative hypothesis, i.e., $K_{1}$ is affected by the quantities of the two Eudragits. According to results, the alternative hypothesis $\mathrm{H} 1$ was accepted, the quantities of the two Eudragits have a significant effect on $K_{1}$. (Tables 3 and 4). The Bayesian estimation of the parameters of the selected model was completed based on the posterior normal distribution of the estimate. The Markov chain has stabilized as indicated by the trace plot (Fig. 2). The posterior distribution of the parameter of the best model $\left(K_{1}\right)$ was normal for all formulations. The estimated K1 varied from 0.006 to 0.012 and it SE from 0.0001 to 0.001 . The SE of the model was 6.154 based on WAIC and 6.4 based on LOO, which confirms the strength of the results. LOO

Table 2. The Rsq (Adjust) and AIC for the release kinetics of Barbital using different mathematical models obtained by DD-Solver.

\begin{tabular}{|c|c|c|c|c|c|c|c|c|c|c|c|c|c|c|}
\hline \multicolumn{2}{|c|}{ Parameters } & \multirow{2}{*}{$\frac{\text { F1 }}{0.89}$} & \multirow{2}{*}{$\frac{\text { F2 }}{-0.74}$} & \multirow{2}{*}{$\frac{\text { F3 }}{-0.32}$} & \multirow{2}{*}{$\begin{array}{c}\text { F4 } \\
0.78\end{array}$} & \multirow{2}{*}{$\frac{\text { F5 }}{0.66}$} & \multirow{2}{*}{$\begin{array}{c}\text { F6 } \\
0.93\end{array}$} & \multirow{2}{*}{$\begin{array}{c}\text { F7 } \\
0.87\end{array}$} & \multirow{2}{*}{$\begin{array}{c}\text { F8 } \\
0.59\end{array}$} & \multirow{2}{*}{$\begin{array}{c}\text { F9 } \\
0.72\end{array}$} & \multirow{2}{*}{$\begin{array}{l}\text { F10 } \\
0.89\end{array}$} & \multirow{2}{*}{$\begin{array}{l}\text { F11 } \\
0.93\end{array}$} & \multirow{2}{*}{$\frac{\text { F12 }}{-0.37}$} & \multirow{2}{*}{$\frac{\text { F13 }}{-0.65}$} \\
\hline Zero order & $R^{2}$ adjust & & & & & & & & & & & & & \\
\hline & K0 & 0.37 & 0.51 & 0.52 & 0.39 & 0.46 & 0.41 & 0.44 & 0.47 & 0.35 & 0.34 & 0.34 & 0.52 & 0.55 \\
\hline & AIC & 30.82 & 44.4 & 43.5 & 34.4 & 37.66 & 29.87 & 33.37 & 38.98 & 33.47 & 29.53 & 27.62 & 44.41 & 43.9 \\
\hline \multirow{3}{*}{$\begin{array}{l}\text { First order } \\
\text { model }\end{array}$} & $R^{2}$ djust & 0.99 & 0.92 & 0.97 & 0.96 & 1 & 0.97 & 0.98 & 0.97 & 0.95 & 0.96 & 0.98 & 0.91 & 0.97 \\
\hline & $\mathrm{K} 1$ & 0.01 & 0.02 & 0.02 & 0.01 & 0.01 & 0.01 & 0.01 & 0.01 & 0.01 & 0.01 & 0.01 & 0.02 & 0.02 \\
\hline & AIC & 16.68 & 28.7 & 23.7 & 25.4 & 15.51 & 25.02 & 24.75 & 25.76 & 24.52 & 24.15 & 21.35 & 30.9 & 23.0 \\
\hline \multirow[t]{3}{*}{ Higuchi model } & $R^{2}$ adjust & 0.94 & 0.61 & 0.77 & 0.92 & 0.96 & 0.91 & 0.91 & 0.9 & 0.98 & 0.93 & 0.92 & 0.64 & 0.78 \\
\hline & $\mathrm{KH}$ & 4.84 & 7.03 & 7.11 & 5.02 & 6.14 & 5.28 & 5.63 & 6.17 & 4.58 & 4.34 & 4.32 & 7.12 & 7.56 \\
\hline & AIC & 28.11 & 36.9 & 34.84 & 29.5 & 27.28 & 31.59 & 31.47 & 31.97 & 19.91 & 27.15 & 28.74 & 37.67 & 33.8 \\
\hline \multirow{4}{*}{$\begin{array}{l}\text { Korsmeyer- } \\
\text { peppas }\end{array}$} & $R^{2}$ adjust & 0.99 & 0.7 & 0.8 & 0.93 & 0.95 & 0.98 & 0.96 & 0.87 & 0.99 & 0.98 & 0.99 & 0.64 & 0.91 \\
\hline & $\mathrm{K}$ & 1.93 & 16.5 & 13.72 & 2.74 & 4.84 & 1.53 & 2.19 & 5.04 & 3.34 & 1.72 & 1.32 & 14.44 & 16.5 \\
\hline & $\mathrm{n}$ & 0.68 & 0.33 & 0.37 & 0.62 & 0.55 & 0.75 & 0.69 & 0.54 & 0.56 & 0.69 & 0.74 & 0.36 & 0.34 \\
\hline & AIC & 21.31 & 36.2 & 34.66 & 29.5 & 28.6 & 24.08 & 28.47 & 33.77 & 18.48 & 22.03 & 19.79 & 38.25 & 30.2 \\
\hline \multirow{3}{*}{$\begin{array}{l}\text { Hixson- } \\
\text { crowoll }\end{array}$} & $R^{2}$ adjust & 0.99 & 0.89 & 0.96 & 0.94 & 0.98 & 0.89 & 0.99 & 0.96 & 0.91 & 0.95 & 0.98 & 0.9 & 0.95 \\
\hline & $\mathrm{KHC}$ & 0 & 0.05 & 0.05 & 0.02 & 0.03 & 0.02 & 0.02 & 0.03 & 0.02 & 0.02 & 0.02 & 0.05 & 0.06 \\
\hline & AIC & 19.68 & 30.6 & 26.41 & 27.5 & 22.37 & 19.3 & 20.26 & 27.57 & 27.92 & 25.22 & 21.72 & 31.5 & 26.2 \\
\hline
\end{tabular}

$\mathbf{A I C}=$ The Akaike Information Criterion 


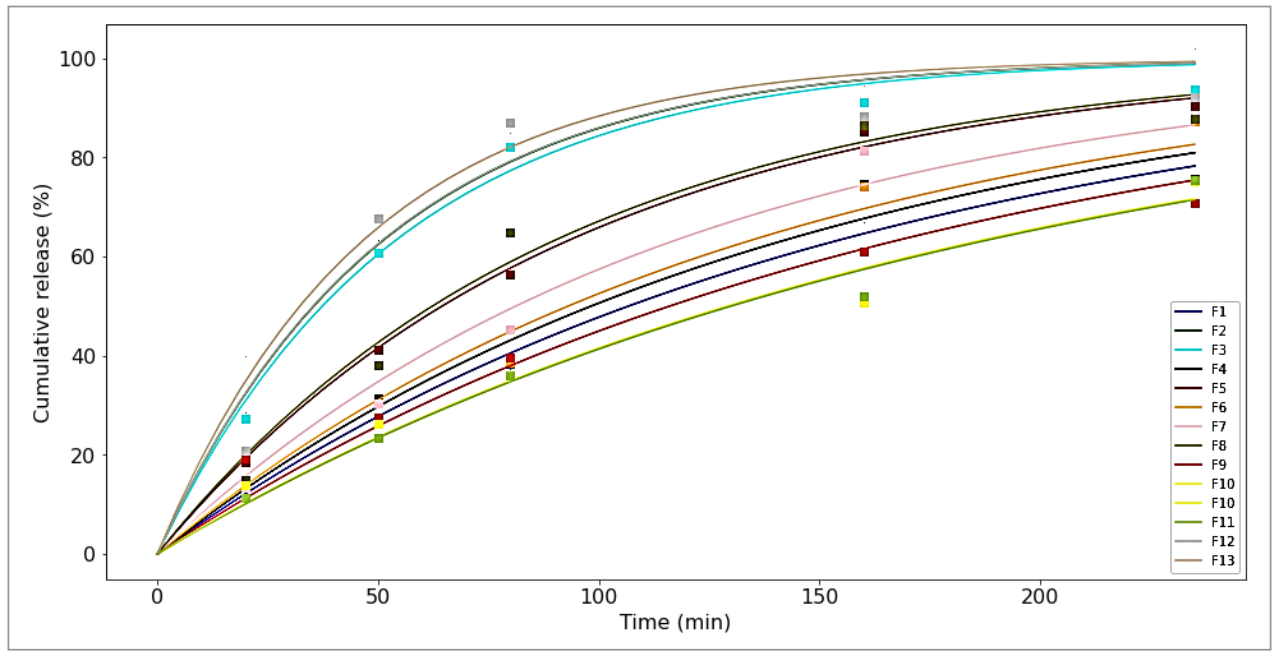

Figure 1. First order kinetic release of Barbital for all formulation.

Table 3. Model comparaison based on WAIC, pWAIC (the effective number of parameters), dWAIC (the difference between the WAIC scores of the other models with the best model), weight (the relative probability that the model explains the data).

\begin{tabular}{lcccccc}
\hline & Rank & WAIC & se & p_waic & d_waic & weight \\
\hline First_Order_hierarchical & 0 & -169.64 & 6.15 & 11.92 & 0.00 & 0.97 \\
Higuchi_hierarchical & 1 & -191.57 & 17.93 & 17.44 & 21.93 & 0.01 \\
Korsemyer_hierarchical & 2 & -196.96 & 15.17 & 23.02 & 27.32 & 0.02 \\
First_Order_pooled & 3 & -203.46 & 8.95 & 5.13 & 33.83 & 0.00 \\
Zero_order_hierarchical & 4 & -240.27 & 12.16 & 28.97 & 70.64 & 0.00 \\
Hixson_hierarchical & 5 & -272.72 & 2.97 & 0.34 & 103.08 & 0.00 \\
\hline
\end{tabular}

Table 4. LOO Statistics of models comparison, p_loo, the effective number of parameters; d_loo (the difference between the LOO scores of the other models with the best model), weight (the relative probability that the model explains the data).

\begin{tabular}{|c|c|c|c|c|c|c|}
\hline & Rank & Loo & se & p_loo & d_loo & weight \\
\hline First_Order_hierarchical & 0 & -170.08 & 6.41 & 12.36 & 0.00 & 0.99 \\
\hline Higuchi_hierarchical & 1 & -192.76 & 16.97 & 18.63 & 22.68 & 0.01 \\
\hline Korsemyer.P_hierarchical & 2 & -199.53 & 13.48 & 25.59 & 29.45 & 0.00 \\
\hline First_Order_pooled & 3 & -203.46 & 8.66 & 5.13 & 33.38 & 0.00 \\
\hline Zero_order_hierarchical & 4 & -244.76 & 10.43 & 33.45 & 74.68 & 0.00 \\
\hline Hixon C_hierarchical & 5 & -272.72 & 3.03 & 0.34 & 102.64 & 0.00 \\
\hline
\end{tabular}

and WAIC estimated the same predictive performance criteria and were asymptotically equal (Gelman et al., 2014; Vehtari et al., 2016; Vehtari and Ojanen, 2012).

The hierarchical method gives a single stable model (First-order model) for the same series of formulations whereas the standard method (frequentist approach) gives several models. The $K_{1}$ of the first-order model estimated by frequentist and Bayesian method were strongly significantly related (Fig. 3). The Bayesian method gave a good estimate of observed data (Fig. 4) and was used for further analysis (VanderPlas, 2014). The relationship between $K_{1}$ and the quantities of the two Eudragits was quadratic (Equation 10). The release rate $\left(K_{1}\right)$ was low for Eudragit L values up to $0.5 \mathrm{~g}(2.5 \%)$ and increases until it reaches its maximum at $2 \mathrm{~g}(10 \%)$ of this Eudragit, on the contrary for Eudragit S, $K_{1}$ decrease from $0 \mathrm{~g}$ to reach it minimum at $1.5 \mathrm{~g}$ (7.5\%) (Fig. 5).
$Y=0.0031 X^{2}-0.0026 X+0.0069(\operatorname{Req}=0.8214)$

with $K=-2 b / a=02 * 0.0026 / 0.0031=0.41$

By this equation which relates the rate of dissolution to the quantity of EL-100, and therefore the mixing of the two Eudragits (total $=10 \%$ ). Drug release from suppository bases generally depends upon the drug solubility in the base and chemical composition of the base. Barbital is a drug in the sodium salt form with a high affinity for hydrophilic bases, i.e., PEG 6000. PEG bases are also known to have a solubility effect, which may partly explain the higher drug release rates of t20 minutes formulations. Both Eudragits create a swellable matrix through which water-soluble active ingredients are released. The mixture advantage became in the difference in solubility of the two polymers in a range of $\mathrm{pH}=7$ to 8 , i.e., with EL-100 Intestinal soluble-fluid from (pH 6) and ES-100 is intestinal soluble-fluid 


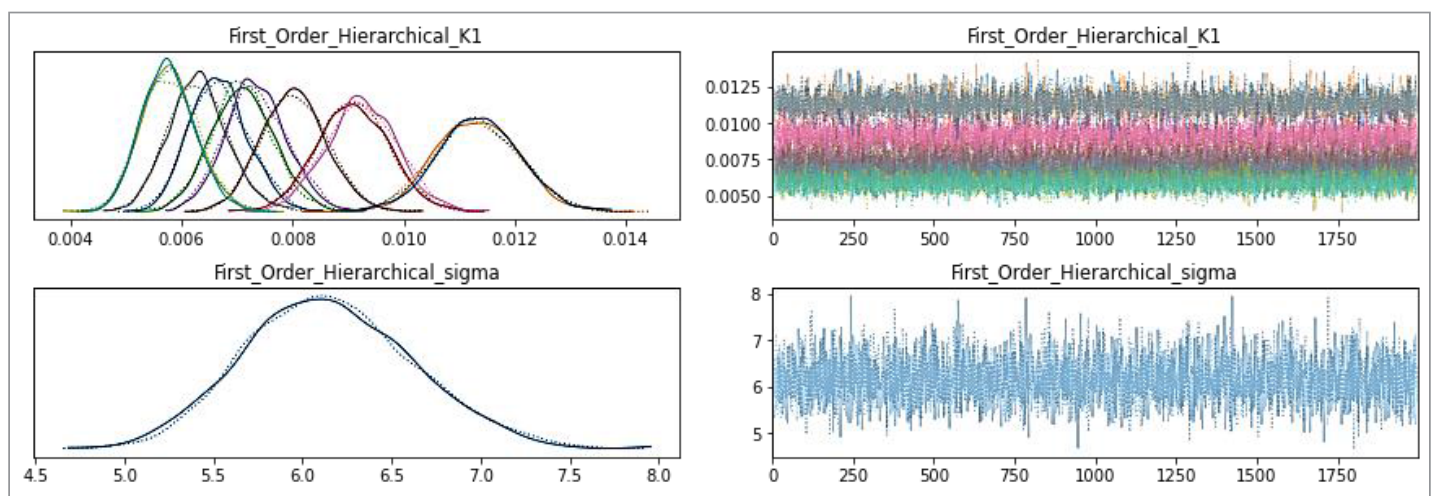

Figure 2. Trace plot and posterior distribution of the the best model parameter (K1) and it standard deviation (sigma).

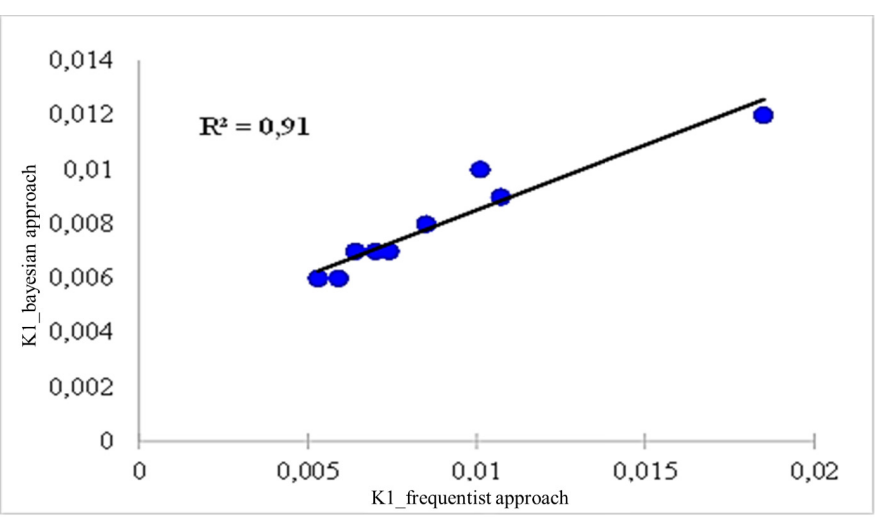

Figure 3. K1 estimated by Bayesian method plotted against K1 estimated by standard method. ( $p$ value an coefficient of determination (R2) were computed in Scipy using least-squares nonlinear regression technique).

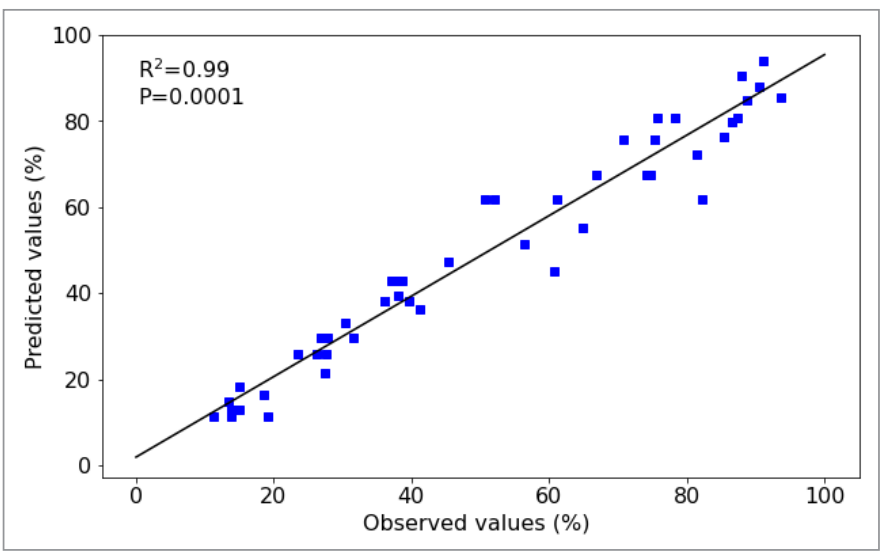

Figure 4. Observed cumulative release plotted against predicted values by the bayesian method ( $p$ value an coefficient of determination (Rsq) were computed in Scipy using least-squares linear regression technique).

from (pH 7) (Di Colo et al., 2002; Hong et al., 2013). The firstorder kinetics model as validated by the Bayesian method as the most adequate model to describe and predict the release of active ingredient, this result is due to the dissolved matrix as a function of the $\mathrm{pH}$ of the rectal region (Dash et al., 2010). Gibaldi and Feldman were the first to propose first-order kinetic models of

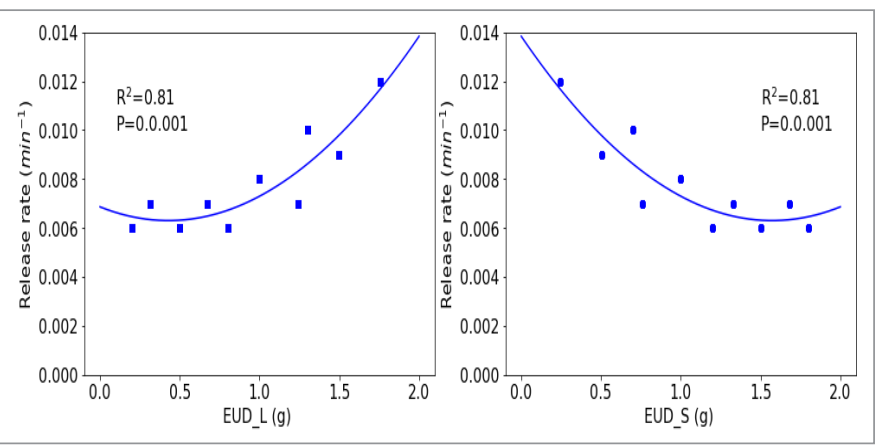

Figure 5. Release rate $\left(\mathrm{K}_{1}\right)$ based on the quantities of two Eudragits L and S. ( $p$ value an coefficient of determination (Rsq) were computed in Scipy using leastsquares linear regression technique).

liberation in 1967 , followed by Wagner in 1969 , for this model the rate of liberation depends on concentration (Costa and Lobo, 2001).

\section{CONCLUSION}

In this study, Bayesian approach was applied to drug release modeling and formulation of sustained-release suppositories containing a mixture of EL-100 and ES-100. This approach allowed finding a unique and the most adequate model, which was the first-order model, when the standard frequentist approach gave three best models. At the same time, the Bayesian approach is a tool for predicting the optimal composition for the formulation of a suppository with prolonged effect. In drug development field, this represented an original and new way to master the kinetics modeling of sustained-release products in order to minimize the number of experiments, save time, and save expensive chemicals.

\section{ACKNOWLEDGMENTS}

We thank Dr. Lahcen Benomar (University of Quebec in Abitibi-Temiscamingue) for his help with statistical analyses. Special thanks are also extended to Dr. Mohamed Taha Moutaoufik (University of Regina) and Read Elfarjani ( TELUQ University) for their valuable comments and suggestions. We thank the reviewers and the editor for relevant comments that helped to improve the content of this article. 


\section{AUTHOR CONTRIBUTIONS}

All authors made substantial contributions to conception and design, acquisition of data, or analysis and interpretation of data; took part in drafting the article or revising it critically for important intellectual content; agreed to submit to the current journal; gave final approval of the version to be published; and agree to be accountable for all aspects of the work. All the authors are eligible to be an author as per the international committee of medical journal editors (ICMJE) requirements/guidelines.

\section{FUNDING}

There is no funding to report.

\section{CONFLICTS OF INTEREST}

The authors report no financial or any other conflicts of interest in this work.

\section{ETHICAL APPROVALS}

This study does not involve experiments on animals or human subjects.

\section{PUBLISHER'S NOTE}

This journal remains neutral with regard to jurisdictional claims in published

\section{REFERENCES}

Andrews RL, Ansari A, Currim . Hierarchical Bayes versus finite mixture conjoint analysis models: A comparison of fit, prediction, and partworth recovery. J Market Res, 2002; 39(1):87-98.

Basak SC, Kumar ,Ramalingam M. Design and release characteristics of sustained release tablet containing metformin $\mathrm{HCl}$. Rev Brasil Ciências Farmacêuticas, 2008; 44(3):477-83.

Bates BC, Campbell . A Markov chain Monte Carlo scheme for parameter estimation and inference in conceptual rainfall-runoff modeling. Water Resour Res, 2001; 37(4):937-47.

Costa $\mathrm{P}$, Lobo . Modeling and comparison of dissolution profiles. Eur J Pharm Sci, 2001; 13(2):123-33.

Cowles MK, Carlin BP. Markov chain Monte Carlo convergence diagnostics: a comparative review. J Am Stat Assoc, 1996; 91(434):883904.

Craig DQ. The mechanisms of drug release from solid dispersions in water-soluble polymers. Int J Pharma, 2002; 231(2):131-44.

Dash S, Murthy , Nath L, Chowdhury P. Kinetic modeling on drug release from controlled drug delivery systems. Acta Pol Pharm, 2010; 67(3):217-23

Di Colo G, Falchi S, Zambito Y. In vitro evaluation of a system for $\mathrm{pH}$-controlled peroral delivery of metformin. J Control Release, 2002; 80(1-3):119-28.

Faisant N, Siepmann J, Benoit J-P. PLGA-based microparticles: elucidation of mechanisms and a new, simple mathematical model quantifying drug release. Eur J Pharm Sci, 2002; 15(4):355-66.

Gelman A, Hwang J, Vehtari A. Understanding predictive information criteria for Bayesian models. Stat Comput, 2014; 24(6):997-1016.

Gouda R, Baishya H, Qing Z. Application of mathematical models in drug release kinetics of carbidopa and levodopa ER tablets. J Dev Drugs, 2017; 6(02)

Grassi M, Grassi G, Lapasin R, Colombo I. Understanding drug release and absorption mechanisms: a physical and mathematical approach CRC Press, Boca Raton, FL, 2006.

Güneri T, Arici M, Ertan G. Sustained release suppositories of ibuprofen microspheres. FABAD J Pharm Sci, 2004; 29:177-84.
Hong H, Carlin , Shamliyan , Wyman , Ramakrishnan R, Sainfort F, Kane . Comparing Bayesian and frequentist approaches for multiple outcome mixed treatment comparisons. Med Decision Making, 2013; 33(5):702-14.

Hua S. Physiological and pharmaceutical considerations for rectal drug formulations. Front Pharmacol, 2019; 10:1196.

Joshi M. Role of eudragit in targeted drug delivery. Int J Curr Pharm Res, 2013; 5(2):58-62.

Khan MZI, Prebeg Ž, Kurjaković N. A pH-dependent colon targeted oral drug delivery system using methacrylic acid copolymers: I. Manipulation of drug release using Eudragit ${ }^{\circledR}$ L100-55 and Eudragit ${ }^{\circledR}$ S100 combinations. J Control Release, 1999; 58(2):215-22.

Lee SH, Bajracharya R, Min , Han , Park , Han . Strategic approaches for colon targeted drug delivery: an overview of recent advancements. Pharmaceutics, 2020; 12(1):68

Luo Y, Al-Harbi K. Performances of LOO and WAIC as IRT model selection methods. Psychol Test Assess Model, 2017; 59(2):183.

Malekmohammadi S, Hadadzadeh H, Rezakhani S, Amirghofran Z. Design and synthesis of gatekeeper coated dendritic silica/titania mesoporous nanoparticles with sustained and controlled drug release properties for targeted synergetic chemo-sonodynamic therapy. ACS Biomater Sci Eng, 2019; 5(9):4405-15.

Millar RB. Comparison of hierarchical Bayesian models for overdispersed count data using DIC and Bayes' factors. Biometrics, 2009; 65(3):962-9.

Misra A. 2014. Applications of polymers in drug delivery. Shrewsbury, United Kingdom: Smithers Rapra.

Moodley K, Pillay V, Choonara YE, Du Toit LC, Ndesendo VM, Kumar P, Cooppan S, Bawa P. Oral drug delivery systems comprising altered geometric configurations for controlled drug delivery. Int J Mol Sci, 2012; 13(1):18-43.

Nakata M. A numerical evaluation of highly accurate multipleprecision arithmetic version of semidefinite programming solver: SDPAGMP,-QD and-DD. IEEE International Symposium on Computer-Aided Control System Design, IEEE, Denver, CO, 2010.

Natanegara F, Neuenschwander B, Seaman Jr JW, Kinnersley N, Heilmann CR, Ohlssen D, Rochester G. The current state of Bayesian methods in medical product development: survey results and recommendations from the DIA Bayesian Scientific Working Group. Pharm Stat, 2014; 13(1):3-12.

Nikam VK, Kotade K, Gaware V, Dolas R, Dhamak K, Somwanshi S, Khadse A, Kashid V. Eudragit a versatile polymer: a review. Pharmacology, 2011; 1(5):152-64.

Onyeji CO, Adebayo AS, Babalola CP. Effects of absorption enhancers in chloroquine suppository formulations: I: In vitro release characteristics. Eur J Pharm Sci, 1999; 9(2):131-6.

Özgüney I, Özcan İ, Ertan G, Güneri T. The preparation and evaluation of sustained release suppositories containing ketoprofen and Eudragit RL 100 by using factorial design. Pharm Dev Technol, 2007; 12(1):97-107.

Paarakh MP, Jose PA, Setty C, Christoper G. Release kineticsconcepts and applications. Int J Pharm Res Technol, 2018; 8(1):12-20.

Patil A, Huard D, Fonnesbeck CJ. PyMC: Bayesian stochastic modelling in Python. J Stat Softw, 2010; 35(4):1.

Peppas NA, Narasimhan B. Mathematical models in drug delivery: how modeling has shaped the way we design new drug delivery systems. J Control Release, 2014; 190:75-81.

Piray P, Dezfouli A, Heskes T, Frank MJ, Daw ND. Hierarchical Bayesian inference for concurrent model fitting and comparison for group studies. PLoS Comput Biol, 2019; 15(6):e1007043.

Price K, LaVange L. Bayesian methods in medical product development and regulatory reviews. Pharm Stat, 2014; 13(1):1-2

Ranjita S, Kamalinder S. In-vitro release of paracetamol from suppocire suppositories: role of additives. In vitro, 2010; 8(1):57-71. 
Salvatier J, Wiecki TV, Fonnesbeck C. Probabilistic programming in Python using PyMC3. PeerJ Comput Sci, 2016; 2:e55.

Surti N, Mahajan A, Misra A. Polymers in rectal drug delivery. Applications of polymers in drug delivery. Elsevier, Amsterdam, Netherlands, 2020, pp 263-80.

Thakral S, Thakral NK, Majumdar DK. Eudragit ${ }^{\circledR}$ : a technology evaluation. Expert Opin Drug Deliv, 2013; 10(1):131-49.

VanderPlas J. 2014. Frequentism and bayesianism: a python-driven primer. arXiv. [ONLINE] Available at: https://arxiv.org/ abs/1411.5018. [Accessed 20 January 2021].

Varma MV, Kaushal AM, Garg A, Garg S. Factors affecting mechanism and kinetics of drug release from matrix-based oral controlled drug delivery systems. Am J Drug Deliv, 2004; 2(1):43-57.

Vehtari A, Gelman A, Gabry J. 2015 . Efficient implementation of leave-one-out cross-validation and WAIC for evaluating fitted Bayesian models. ArXiv. [ONLINE] Available at: https://arxiv.org/. [Accessed 20 January 2021].

Vehtari A, Gelman A, Gabry J. Practical Bayesian model evaluation using leave-one-out cross-validation and WAIC. Stat Comput, 2017; 27(5):1413-32.

Vehtari A, Mononen T, Tolvanen V, Sivula T, Winther O. Bayesian leave-one-out cross-validation approximations for Gaussian latent variable models. J Mach Learn Res, 2016; 17(1):3581-618.

Vehtari A, Ojanen J. A survey of Bayesian predictive methods for model assessment, selection and comparison. Stat Surv, 2012; 6:142-228.
Victor O, Francis O. Evaluation of the kinetics and mechanism of piroxicam release from lipophilic and hydrophilic suppository bases. Int J Chem Tech Res, 20171 10:189-98

Watanabe S, Opper M. Asymptotic equivalence of Bayes cross validation and widely applicable information criterion in singular learning theory. J Mach Learn Res, 2010; 11(12):3571-94.

Wiecki TV, Sofer I, Frank MJ. HDDM: hierarchical Bayesian estimation of the drift-diffusion model in python. Front Neuroinform, 2013; $7: 14$.

Zhang Y, Huo M, Zhou J, Zou A, Li W, Yao C, Xie S. DDSolver: an add-in program for modeling and comparison of drug dissolution profiles. AAPS J, 2010; 12(3):263-71.

Zuo J, Gao Y, Bou-Chacra N, Löbenberg R. Evaluation of the DDSolver software applications. Biomed Res Int, 2014; 2014:204925.

\section{How to cite this article:}

Benomar A, Adade CA, Elalaoui Y, Cherkaoui N, Rahali Y, Laatiris A, Fahry A. Hierarchical Bayesian approach applied to the formulation of sustained-release suppositories and dissolution profile modeling. J Appl Pharm Sci, 2021; 11(07):055-062. 